\title{
Interference Mitigation Method in Hybrid D2D and Cellular Network
}

\author{
Mohammed H. M. Sadig, and Amin B. A. Mustafa
}

\begin{abstract}
This paper studies the effect of Device-to-Device interference in cellular networks. The focus of this study is the underlay inband interference resulting from sharing the same frequency. We propose a new mechanism that alleviates the effect of the additional interference in this hybrid system. Utilizing this interference mitigation method does not violate the frequency allocation of the cellular network. The quality of the studied network is evaluated using signal to interference plus noise ratio (SINR). The proposed approach reduces the interference levels in the uplink mode by $55 \%$.
\end{abstract}

Index Terms-Cellular network; Device-to-Device; Hybrid System; Interference; SINR; Underlay inband.

\section{INTRODUCTION}

Nowadays, the major challenge that faces both telecom vendors and operators is to satisfy the increasingly high demand of the mobile users. However, the up-to-date technologies such as $4 \mathrm{G}$ are very efficient and pushed to their limits, yet to some point, these technologies cannot undertake the users' drastic need. Accordingly, the scholars and researchers are continuously in a hunt for new communication techniques to evolve the conventional cellular network. Device-to-Device (D2D) communication can enhance the performance of today's cellular network and this paradigm is expected to play a vital role in the upcoming $5 \mathrm{G}$ generation. [1]

The Devise-to-Devise communication (D2D) as the name implies, it is the paradigm where the source of information can directly communicate with the destination, without relying on any third party or intermediate node. $\mathrm{WiFi}$ and Bluetooth can be considered as examples for D2D communication networks. However, in the case of the cellular and mobile network, D2D means that the User Equipment will directly communicate with each other without the intervention of the base station if the users are close enough to each other.

Generally, many papers proposed different categorizations for the ways by which the hybrid D2D Cellular system is set. The authors in [2] pointed out four setups; based on the presence and absence of the BS control, and also based on whether the device is directly communicating with another devise, or just relaying on it. Hence the types are Device relaying with operator controlled link establishment (DR-OC), Direct D2D communication with operator controlled link establishment

Published on December 29, 2017

Mohammed H. Sadig is a lecturer at Bayan College for Science \& Technology, Alslama College, and Cambridge International College, Sudan. (e-mail: mhyder09@live.com).

Dr. Amin B. Mustafa is with the Electrical Engineering Department, Alneelain University (amin31766@gmail.com)
(DC-OC), Device relaying with device controlled link establishment (DR-DC) and finally Direct D2D communication with device controlled link establishment (DC-DC). Furthermore, the authors in [1], categorized the hybrid system upon the way how the frequency is shared between the D2D and the cellular network and the types are mainly Inband and Outband and the Inband is further divided into Overlay Inband and Underlay Inband as shown obviously in Fig 1. According to the specified categories in this paper we will focus on a system where the devices are directly communicating with other, and link control is from the base station (i.e. DC - OC) as shown in Fig. 2, and the spectrum is shared between the cellular and D2D (i.e. Inband Underlay)

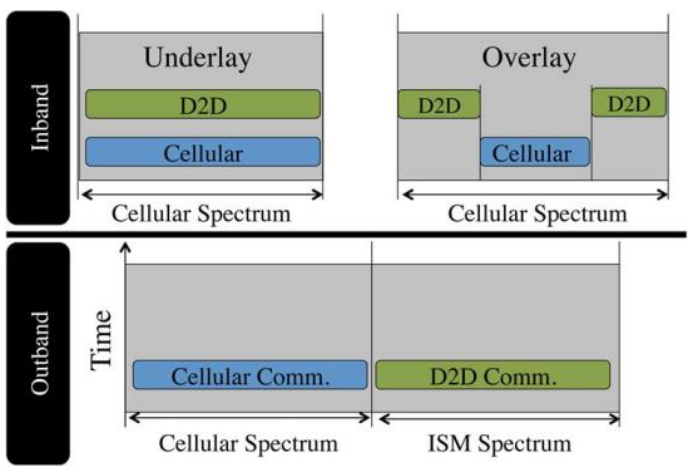

Fig. 1. Schematic representation of overlay inband, underlay inband, and outband D2D.[1]

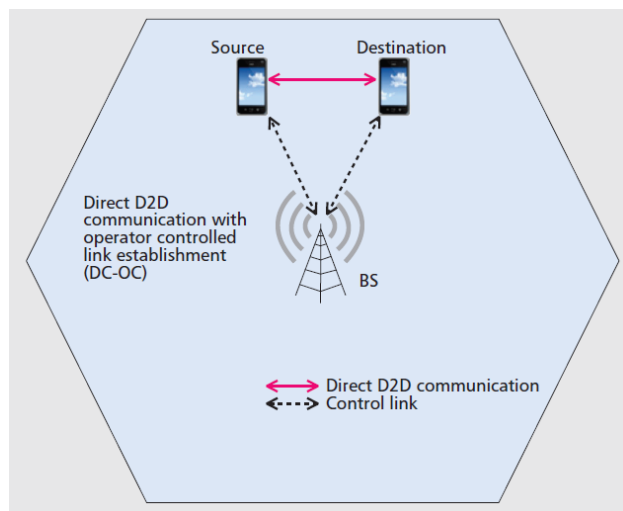

Fig. 2. Direct D2D communication with operator controlled link establishment (DC-OC) [2]

Exploiting direct communication between nearby mobile devices will improve the spectrum utilization, overall throughput and energy efficiency. On the other hand, D2D will also give rise to major challenges such as device design, security issues, mobility management and interference management. The effect of the interference is more noticeable in the underlay Inband.

To reduce the Interference Brett Kaufman and Behnaam 
Aazhang [3] proposed an approach by which the D2D devices receive a signal from the BS through the downlink control channel, and then make an estimate for the path loss between the D2D transmitter and the BS. Using this estimate the power of the D2D transmitter will always be below some threshold so that the interference to the cellular uplink is reduced as the power of the D2D transmitter is reduced.

The authors in [4] consider the Interference underlay Inband mode and focus on the Uplink LTE scenario and propose two mechanisms to mitigate the interference between the D2D and the cellular network. One mechanism is the Interference tracking approach by avoiding the interference coming from the cellular network to the D2D network and the other mechanism is the Interference broadcasting approach, to avoid the interference coming from the D2D network to the cellular network.

In this paper, we will introduce a mechanism similar to method used in [3] and the Interference broadcasting approach used in [4]; where we focus on mitigating the interference coming from the D2D users to the cellular network.

The next sections of this paper will introduce our proposed approach in section II which describes our model in general, and shows the measure of the interference in three different scenarios. Section III shows the results and comparisons for the three cases and section IV concludes our work.

\section{PROPOSED APPROACH}

In this section we will propose three different scenarios; Scenario I only cellular users were considered, Scenario II hybrid system (i.e. Cellular + D2D users) with a random frequency allocation and distribution for both cellular and D2D devices, and Scenario III hybrid system with random frequency allocation and controlled distribution for D2D devices. In each scenario we will compute the Signal to Interference plus Noise Ratio (SINR).

The channel model used for both cellular and D2D links is a general Rayleigh faded and Additive White Gaussian Noise AWGN. This model can be expressed with a received signal (r) and Rayleigh RV (h) and transmitted signal (s) and Gaussian RV (n) as the following:

$r=h * s+n$

\section{A. Scenario I}

In this scenario we assumed a seven-cells cluster for our cellular network. Mobile users were scattered randomly with a uniform distribution. Generally, the users from the cells that are using the same frequency band used by our cell of interest will cause interference as shown in Fig. 3.

The SINR for each link in the uplink model is then to be computed according to the channel model using the Rayleigh RV, UE power (p), distance from the BS (d), distance from the interfering user i to the BS (di), path loss exponent (L) noise variance (N0) and a channel bandwidth (B) as follows:
$\operatorname{SINR}=\frac{h * P * d^{-L}}{B * N_{0}+\sum_{i=1}^{6} h * P * d_{i}^{-L}}$

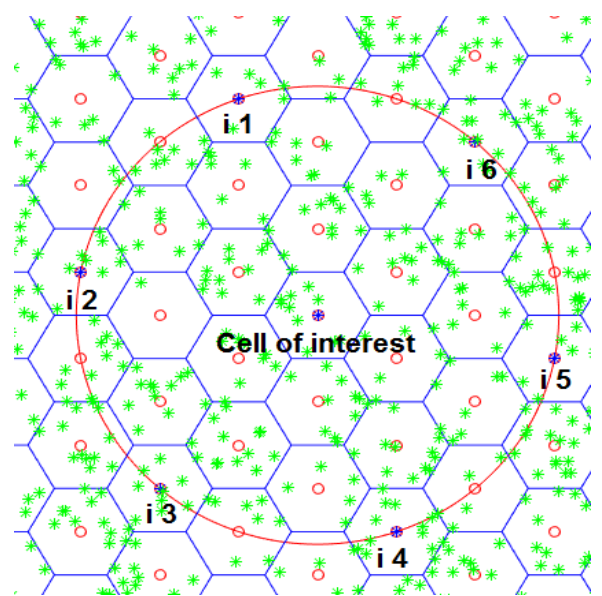

Fig.3. Seven-cells Cluster with six interfering cell for each cell (Scenario I)

\section{B. Scenario II}

We will add D2D devices to the previous plain cellular network conditioning that the number of D2D users is twice the number of the cellular network users, which gives a worst case situation; when all the channels used for the cellular network will be reused by the D2D users, since each two D2D users will share only one channel. The D2D will also be distributed randomly, as illustrated in Fig. 4 same previous equation but it is important to address the interference resulting from the D2D devices with a distance (Dd) between the D2D transmitter and the BS and distance (Ddi) between the D2D transmitter in the interfering cells, and hence the SINR will be as follows:

$\operatorname{SINRd}=\frac{h * P * d^{-L}}{B * N_{0}+h * P * D d^{-L}+\sum_{i=1}^{6} h * P * d_{i}^{-L}+\sum_{i=1}^{6} h * P * D d_{i}^{-L}}$

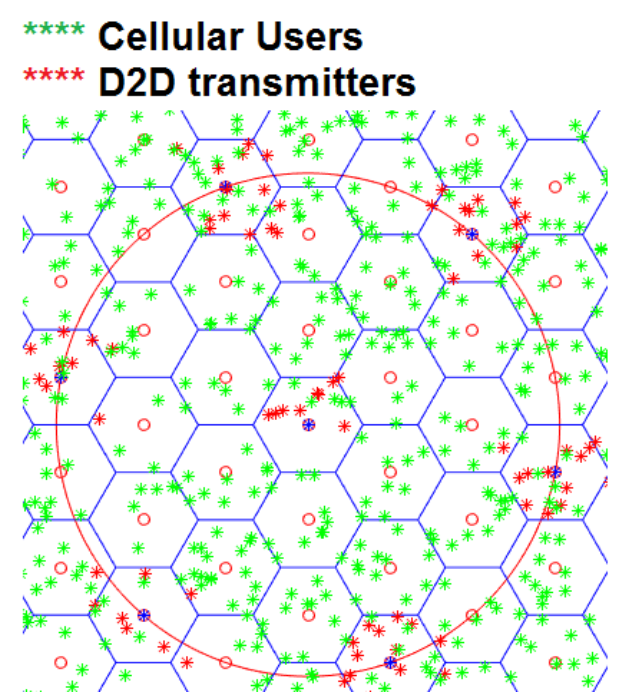

Fig.4. Hybrid D2D and Cellular network with non-controlled random distribution for the D2D devices (Scenario II)

\section{Scenario III}

Here we proposed a controlled distribution for the D2D devices within the network. This new distribution governs the D2D devise to be scattered outside a circle with some radius $r$ to ensure that their contribution in the interference equation is always less than the desired signal power (i.e. 
Dd $>$ d). Such a kind of controlled distribution is shown in Fig 5.

SINR equation is the similar to the equation in the second scenario, but for sure the distances measures will change.

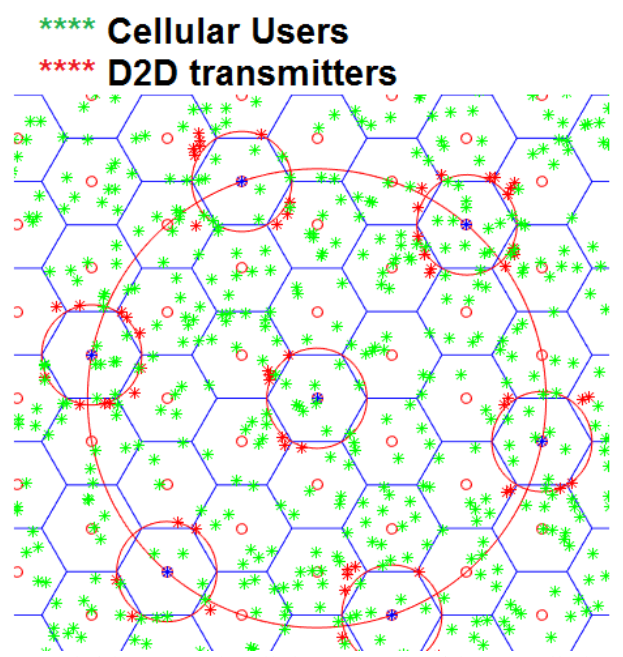

Fig. .5. Hybrid D2D and Cellular network with controlled random distribution for the D2D devices (Scenario III)

To obtain numerical results for the SINR, we made assumptions for the values used in the equation. Table I shows these values.

As long as in our approach random variables were used, therefore, we applied Monte-Carlo computational algorithm for many Iterations to give an estimate for the average SINR value

TABLE I: SYSTEM ASSUMPTIONS

\begin{tabular}{cc} 
TABLE I: SYSTEM ASSUMPTIONS \\
\hline \hline User power (P) & $25 \mathrm{mWatt}$ \\
Noise $\left(\mathrm{N}_{0}\right)$ & $-170 \mathrm{dBm}$ \\
The channel BW (B) & $200 \mathrm{kHz}$ \\
Path loss exponent (L) & 3 \\
Number of cells per Cluster (N) & 7 \\
\hline \hline
\end{tabular}

\section{RESULTS}

In this section we will view the results for the three scenarios; plain cellular network, Hybrid System with random distribution and results for the Hybrid System with controlled distribution for the D2D users. Table II shows the average SINR for the three scenarios and the controlled distribution enhanced the SINR by $55 \%$

TABLE II: AVERAGE SINR FOR THE THREE SCENARIOS

\begin{tabular}{cc}
\hline \hline Scenario & Avg SINR \\
\hline Cellular Network (I) & $16.7747 \mathrm{~dB}$ \\
Hybrid non-controlled Random Distribution & $9.0982 \mathrm{~dB}$ \\
Hybrid Controlled Random Distribution & $14.5627 \mathrm{~dB}$ \\
\hline \hline
\end{tabular}

\section{A. Scenario I}

The graph in Fig. 6 shows the result of the SINR for the first ten iterations where the interference is coming only from the cellular users

\section{B. Scenario II}

In the second case when introducing the D2D without controlling the distribution as can be noticed from the graph in Fig. 7 the values of the SINR are relatively low when compared with the cellular system.

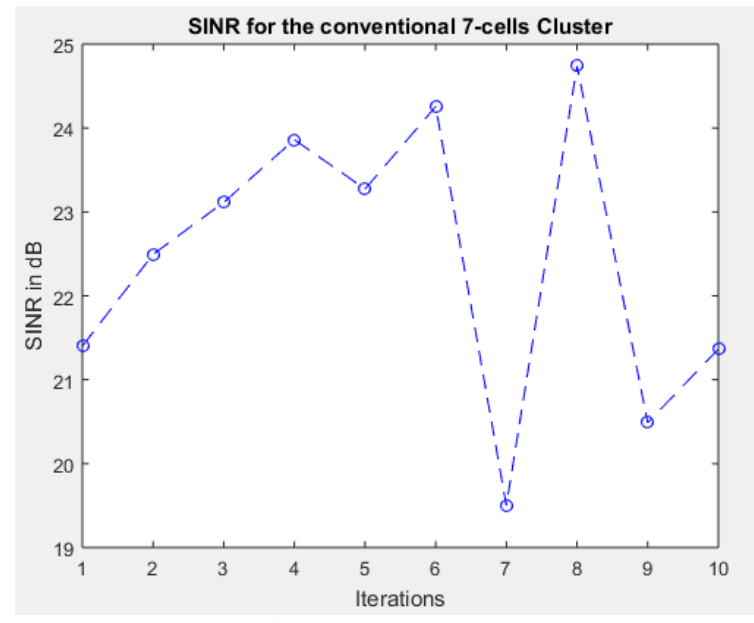

Fig. 6. SINR for the conventional 7-cells cluster

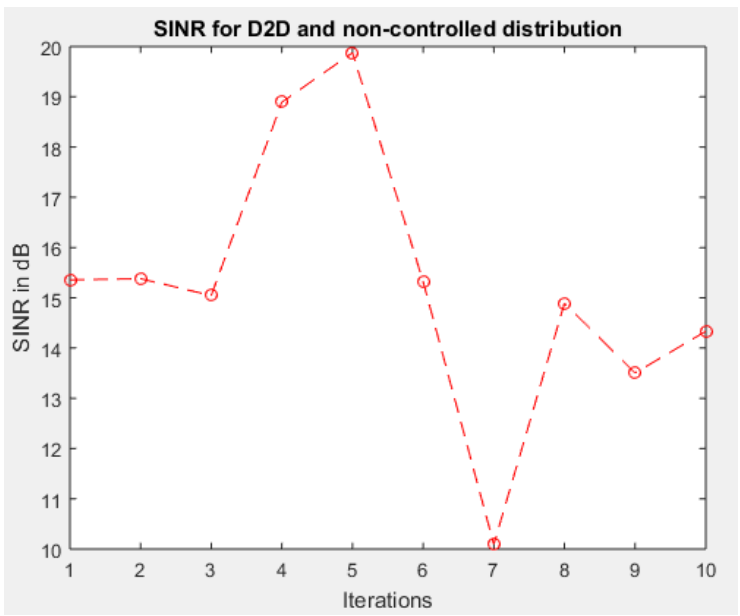

Fig .7. SINR for D2D and non-controlled distribution.

\section{Scenario III}

This scenario as expected increased the SINR values when limiting the D2D users to be active within predefined region. The results in Fig. 8. shows this case

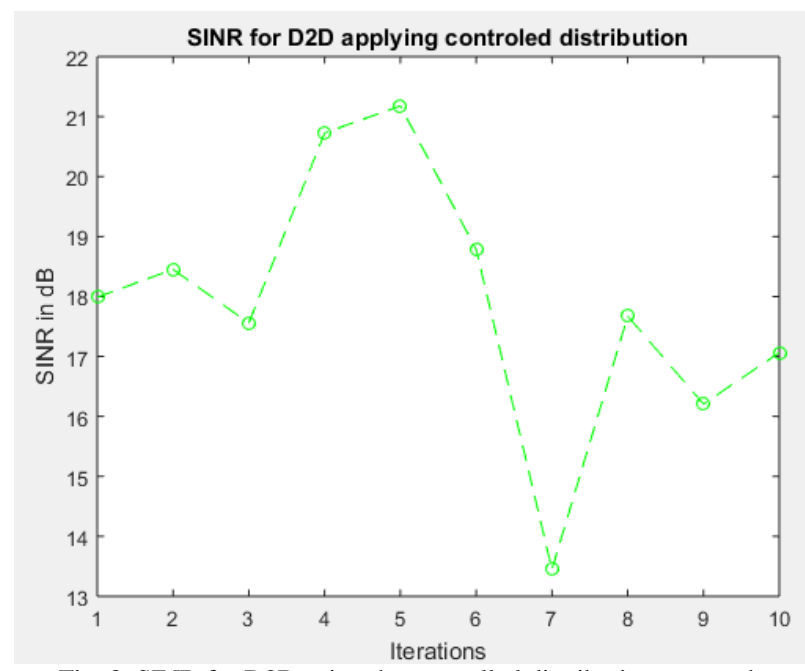

Fig. 8. SINR for D2D using the controlled distribution approach

Furthermore, as a comprehensive comparison in Fig. 9 where it is obvious that the SINR profiles for both 
controlled and non-controlled distribution are similar to the SINR profile for the cellular network before adding the D2D network. This observation implies that if the cellular network is insusceptible to interference it will give a better performance after adding the D2D regardless the allocation of the D2D and vice versa.

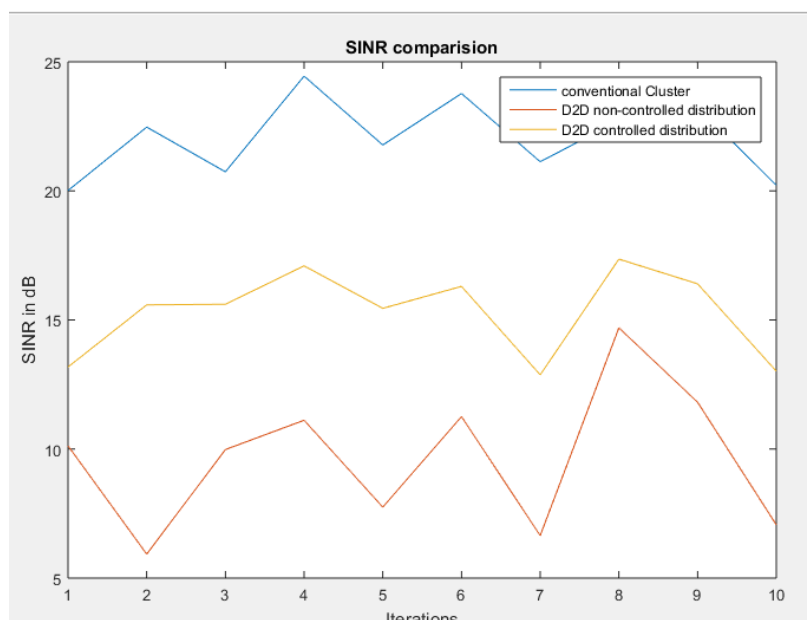

Fig. 9. The SINR for the three scenarios VS the Iterations

\section{CONCLUSION}

The interference from the D2D to cellular network in the underlay inband is impossible to eliminate, but, however a method to reduce its effect is efficiently proposed by this paper.

\section{REFERENCES}

[1] A. Asadi, Q. Wang, and V. Mancuso, "A survey on device-to-device communication in cellular networks," IEEE Communications Surveys \& Tutorials, vol. 16, no. 4, pp. 1801-1819, 2014.
[2] Tehrani, M.N., Uysal, M. and Yanikomeroglu, H. (2014) 'Device-todevice communication in $5 \mathrm{G}$ cellular networks: Challenges, solutions, and future directions', IEEE Communications Magazine, 52(5), pp. 86-92. doi: 10.1109/mcom.2014.6815897.

[3] B. Kaufman and B. Aazhang, "Cellular networks with an overlaid device to device network," in Proc. Asilomar Conf. Signals, Syst. Comput., 2008, pp. 1537-1541.R.

[4] T. Peng, Q. Lu, H. Wang, S. Xu, and W. Wang, "Interference avoidance mechanisms in the hybrid cellular and device-to-device systems," in Proc. IEEE PIMRC, 2009, pp. 617

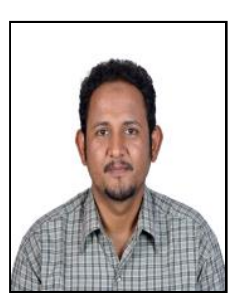

Mohammed Hyder Mekki Sadig has obtained his B.Sc. degree in Electrical and Electronic Engineering from Bayan College for Science \& Technology in 2009.

He has obtained his MSc. degree from Alzaiem Alazhari Univesity Electrical and Electronic Engineering department in 2014. He has been a PhD student Since then 2105 .

Mohamed worked and still working as lecturer in Bayan College for Science \& Technology, Alslam College, and Cambridge International College (Sudan) and supervised many final year graduation projects.

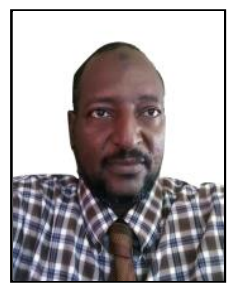

Associate Prof. Dr. Amin Babiker A/Nabi Mustafa has obtained his B.Sc. degree in Electrical and Electronic Engineering from Faculty of Engineering University of Khartoum in 1990.

He has obtained his $\mathrm{Ph} . \mathrm{D}$. degree from Alneelain University Faculty of Engineering in 2007. He has been the Dean, Faculty of Engineering Alneelain University since 2009 up to 2015 when he has taken the post of Secretary of Academic Affairs.

Prof Amin Supervised or Supervising more than 150 M.Sc. or Ph.D. Students. Dr. Amin has been appointed as External Examiner for so many Universities. He is a member of Sudan Engineering Council, Federation of Sudanese Engineers, Federation of Arab Engineers and its Engineering Education Committee.

Dr. Amin Published over 300 articles in International, Regional and Local Scientific Journals. He participated in several related international conferences. He has been the Co-chair of IEEE Conference organized by Alneelain University in 2015. 